\title{
OPEN Propensity score-adjusted analysis on stent-assisted coiling versus coiling alone for ruptured intracranial aneurysms
}

\author{
Lukas Goertz ${ }^{1,2 \bowtie}$, Thomas Liebig ${ }^{3}$, Lenhard Pennig ${ }^{1}$, Marco Timmer $^{1}$, Hanna Styczen ${ }^{4}$, \\ Jan-Peter Grunz ${ }^{5}$, Thorsten Lichtenstein ${ }^{1}$, Marc Schlamann ${ }^{1}$ \& Christoph Kabbasch ${ }^{1}$
}

Stent-assisted coiling (SAC) for ruptured intracranial aneurysms (RIAs) remains controversial due to an inherent risk of potential thromboembolic and hemorrhagic complications. We compared SAC and coiling alone for the management of RIAs using propensity score-adjustment. Sixty-four patients treated by SAC and 220 by stand-alone coiling were retrospectively reviewed and compared using inverse probability of treatment weighting (IPTW) with propensity scores. Functional outcome, procedure-related and overall complications and angiographic results were analyzed. Aneurysms treated by SAC had a larger diameter, a wider neck and were more frequently located at the posterior circulation. SAC had a higher risk for thromboembolic complications $(17.2 \%$ vs. $7.7 \%, p=0.025)$, however, this difference did not persist in the IPTW analysis (OR 1.2, 95\% CI 0.7-2.3, adjusted $p=0.458)$. In the adjusted analysis, rates of procedural cerebral infarction $(p=0.188)$, ventriculostomyrelated hemorrhage $(p=0.584)$, in-hospital mortality $(p=0.786)$ and 6 -month favorable functional outcome ( $p=0.471$ ) were not significantly different between the two groups. SAC yielded a higher complete occlusion $(80.0 \%$ vs. $67.2 \%$, OR $3.2,95 \% \mathrm{Cl} 1.9-5.4, \mathrm{p}<0.001)$ and a lower recanalization rate ( $17.5 \%$ vs. $26.1 \%$, OR $0.3,95 \% \mathrm{Cl} 0.2-0.6, \mathrm{p}<0.001)$ than stand-alone coiling at 6 -month follow-up. In conclusion, SAC of large and wide-necked RIAs provided higher aneurysm occlusion and similar clinical outcome, when compared to stand-alone coiling.

Endovascular coil embolization represents the standard treatment modality for intracranial aneurysms. The adjunct use of intracranial stents prevents coil protrusion into the parent artery and facilitates coiling of widenecked aneurysms with an unfavourable dome-to-neck ratio ${ }^{1,2}$. Since stent-assisted coiling (SAC) allows for a denser coil packing, it provides better long-term aneurysm occlusion than balloon-assisted coiling or coiling alone $^{3,4}$. Procedural complications of SAC are mainly attributable to thromboembolic and hemorrhagic events because of the mandatory anti-platelet therapy ${ }^{5}$. Particularly for the latter reason, the use of SAC for acutely ruptured aneurysms remains controversial. There is concern that double anti-platelet medication might increase the risk of aneurysm rebleeding and can complicate intracranial surgical procedures which might be necessary during the acute phase of subarachnoid hemorrhage $(\mathrm{SAH})^{6,7}$. On the contrary, insufficient anti-platelet therapy may increase the risk of cerebral infarction, which is a major cause for treatment-related morbidity. Nevertheless, previous studies have provided evidence that SAC combined with state-of-the-art antiplatelet therapy can be safe and effective for the management of acutely ruptured aneurysms $\mathrm{s}^{8-10}$. Moreover, a recent meta-analysis by Zhang et al. reported similar functional outcome and a lower recurrence rate for SAC when compared to stand-alone coiling ${ }^{11}$. However, since SAC is generally used for more complex aneurysms, a direct comparison between the treatment modalities is impeded. To date, there have been no prospective randomized studies comparing

\footnotetext{
${ }^{1}$ Center for Neurosurgery, Medical Faculty and University Hospital, University of Cologne, Kerpener Strasse 62, 50937 Cologne, Germany. ${ }^{2}$ Department of Neuroradiology, Medical Faculty and University Hospital, University of Cologne, Kerpener Strasse 62, 50937 Cologne, Germany. ${ }^{3}$ Department of Neuroradiology, LMU University Hospital of Munich, Marchioninistraße 15, 81377 Munich, Germany. ${ }^{4}$ Institute for Diagnostic and Interventional Radiology and Neuroradiology, University Hospital Essen, Hufelandstraße 55, 45147 Essen, Germany. ${ }^{5}$ Department of Diagnostic and Interventional Radiology, University Hospital Wuerzburg, Josef-Schneider-Straße 2, 97080 Wuerzburg, Germany. ${ }^{\circledR}$ email: lukas.goertz@uk-koeln.de
} 


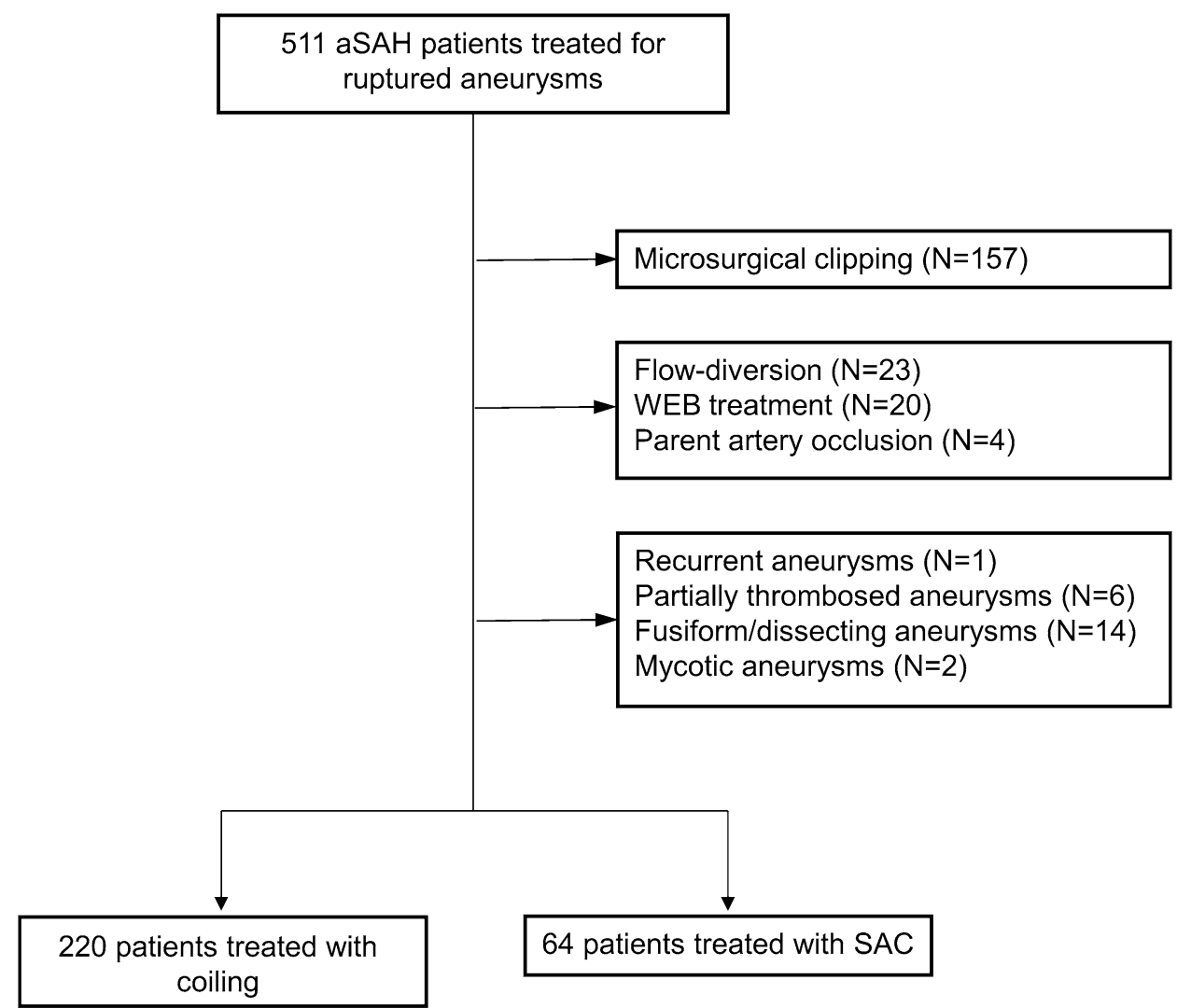

Figure 1. Flow chart of patient selection. aSAH, aneurysmal subarachnoid hemorrhage; WEB, Woven Endobridge; SAC, stent-assisted coiling.

SAC to coiling alone and no comparative studies that systematically adjust for diverging baseline patient and aneurysm characteristics.

The objective of the current study was to compare SAC and coiling alone for ruptured intracranial aneurysms regarding procedural complications, clinical outcome, and angiographic results. In order to create homogenous study groups, retrospective randomization was performed using inverse probability treatment weighting (IPTW) based on individual propensity scores.

\section{Results}

Patient and aneurysm characteristics. During the study period, 511 patients were treated for a ruptured aneurysm. Thereof, 284 patients met the inclusion criteria and were enrolled. Patient selection is detailed in Fig. 1. The mean patient age was $54.3 \pm 14.0$ years and 183 patients were female $(64.4 \%)$. The ruptured aneurysm was located at the intradural internal cerebral artery in 70 cases $(24.6 \%)$, at the anterior cerebral artery in $127(44.7 \%)$, at the middle cerebral artery in $31(10.9 \%)$ and at the posterior circulation in $56(19.7 \%)$. The mean aneurysm size was $7.5 \pm 3.7 \mathrm{~mm}$ and the mean neck width was $3.3 \pm 1.6 \mathrm{~mm}$. The aneurysms were treated within $48 \mathrm{~h}$ after ictus in 242 cases (85.2\%). Stand-alone coiling was performed in 220 patients (77.5\%), while adjunctive stents were used in $64(22.5 \%)$. In the coiling alone group, 30 patients were treated with balloonassistance (13.6\%). In the SAC group, stent implantation was planned a priori in 53 patients (82.8\%) due to a wide aneurysm neck. In 11 patients (17.2\%), the stent was implanted as a salvage technique, because the coils protruded or tended to protrude into the parent artery $(n=10)$, or to allow a denser coil packing $(n=1)$. Aneurysms treated with the assistance of intracranial stents were more frequently located at the posterior circulation $(39.1 \%$ vs. $14.1 \%, \mathrm{p}<0.001)$, had a larger mean diameter $(8.7 \pm 4.4 \mathrm{~mm}$ vs. $7.2 \pm 3.4 \mathrm{~mm}, \mathrm{p}=0.003)$ and a wider neck $(4.5 \pm 2.2 \mathrm{~mm}$ vs. $3.0 \pm 1.2 \mathrm{~mm}, \mathrm{p}<0.001)$ than aneurysms treated without stent assistance. After IPTW, the two groups were comparable regarding all baseline characteristics as detailed in Table 1.

Immediate aneurysm occlusion. The control angiography scan after the procedure showed complete aneurysm occlusion, neck remnants and aneurysm remnants in $85.5 \%, 9.5 \%$ and $5.0 \%$ for coiling, respectively, and in $87.5 \%, 10.9 \%$ and $1.6 \%$ for SAC, respectively $(\mathrm{p}=0.549)$. After propensity score adjustment, SAC was associated with higher odds for complete aneurysm occlusion (OR 1.9, 95\% CI 1.1-3.2, p=0.018) while standalone coiling carried a higher risk for aneurysm remnants (OR 2.7, 95\% CI 1.1-7.0, p=0.030). 


\begin{tabular}{|c|c|c|c|c|c|c|}
\hline & \multicolumn{3}{|c|}{ Unadjusted analysis } & \multicolumn{3}{|l|}{ IPTW analysis } \\
\hline & Coiling $(n=220)$ & SAC $(n=64)$ & $\mathbf{P}$ & Coiling $(n=287)$ & SAC $(n=251)$ & $\mathbf{P}$ \\
\hline Patient age (years) & $54.0 \pm 14.4$ & $55.5 \pm 12.6$ & 0.441 & $53.7 \pm 14.4$ & $54.2 \pm 10.9$ & 0.669 \\
\hline Gender & & & 0.158 & & & 0.530 \\
\hline Female & $137(62.3 \%)$ & $46(71.9 \%)$ & & $187(65.2 \%)$ & $157(62.5 \%)$ & \\
\hline Male & $83(37.7 \%)$ & $18(28.1 \%)$ & & $100(34.8 \%)$ & $94(37.5 \%)$ & \\
\hline WFNS grade & & & 0.847 & & & 0.231 \\
\hline WFNS 1 & $72(32.7 \%)$ & $20(31.3 \%)$ & & $90(31.4 \%)$ & $80(31.9 \%)$ & \\
\hline WFNS 2 & $42(19.1 \%)$ & $9(14.1 \%)$ & & $48(16.7 \%)$ & $28(11.2 \%)$ & \\
\hline WFNS 3 & $18(8.2 \%)$ & $5(7.8 \%)$ & & $25(8.7 \%)$ & $17(6.8 \%)$ & \\
\hline WFNS 4 & $27(12.3 \%)$ & $10(15.6 \%)$ & & $40(13.9 \%)$ & $35(13.9 \%)$ & \\
\hline WFNS 5 & $61(27.7 \%)$ & $20(31.3 \%)$ & & $84(29.3 \%)$ & $91(36.3 \%)$ & \\
\hline Fisher grade & & & 0.359 & & & 0.262 \\
\hline Fisher 1 & $2(0.9 \%)$ & $1(1.6 \%)$ & & $3(1.0 \%)$ & $6(2.4 \%)$ & \\
\hline Fisher 2 & $15(6.8 \%)$ & $5(7.8 \%)$ & & $21(7.3 \%)$ & $21(8.4 \%)$ & \\
\hline Fisher 3 & $103(46.8 \%)$ & $22(34.4 \%)$ & & $124(43.2 \%)$ & $91(36.3 \%)$ & \\
\hline Fisher 4 & $100(45.5 \%)$ & $36(56.3 \%)$ & & $138(48.1 \%)$ & $134(53.4 \%)$ & \\
\hline IVH & $82(37.3 \%)$ & $33(51.6 \%)$ & 0.040 & $118(41.1 \%)$ & $123(49.0 \%)$ & 0.073 \\
\hline $\mathrm{ICH}$ & $39(17.7 \%)$ & $10(15.6 \%)$ & 0.695 & $47(16.4 \%)$ & $40(15.9 \%)$ & 0.874 \\
\hline \multicolumn{7}{|l|}{ Aneurysm location } \\
\hline ICA & $57(25.9 \%)$ & $13(20.3 \%)$ & 0.361 & $69(24.0 \%)$ & $49(19.5 \%)$ & 0.206 \\
\hline ACA & $106(48.2 \%)$ & $21(32.8 \%)$ & 0.030 & $127(44.3 \%)$ & $128(51.0 \%)$ & 0.120 \\
\hline MCA & $26(11.8 \%)$ & $5(7.8 \%)$ & 0.366 & $32(11.1 \%)$ & $23(9.2 \%)$ & 0.448 \\
\hline $\mathrm{PC}$ & $31(14.1 \%)$ & $25(39.1 \%)$ & $<0.001$ & $59(20.6 \%)$ & $51(20.3 \%)$ & 0.945 \\
\hline Aneurysm size (mm) & $7.2 \pm 3.4$ & $8.7 \pm 4.4$ & 0.003 & $7.7 \pm 4.0$ & $8.1 \pm 3.9$ & 0.315 \\
\hline Neck width (mm) & $3.0 \pm 1.2$ & $4.5 \pm 2.2$ & $<0.001$ & $3.3 \pm 1.6$ & $3.7 \pm 1.5$ & 0.110 \\
\hline Dome-to-neck ratio & $1.9 \pm 0.7$ & $1.8 \pm 1.0$ & 0.310 & $1.9 \pm 0.7$ & $1.8 \pm 0.9$ & 0.071 \\
\hline Aneurysm treatment $<48 \mathrm{~h}$ after ictus & $184(83.6 \%)$ & $58(90.6 \%)$ & 0.166 & $237(82.6 \%)$ & $220(87.6 \%)$ & 0.101 \\
\hline
\end{tabular}

Table 1. Baseline patient and aneurysm characteristics. IPTW, inverse probability of treatment weighting; SAC, stent-assisted coiling; WFNS, World Federation of Neurosurgical Societies grading scale; IVH, intraventricular haemorrhage; ICH, intracranial hemorrhage; ICA, internal carotid artery; ACA, anterior cerebral artery; MCA, middle cerebral artery; PC, posterior circulation.

\begin{tabular}{|l|l|l|l|c|}
\hline & Coiling $(\mathbf{n}=\mathbf{2 2 0})$ & SAC $(\mathbf{n}=\mathbf{6 4})$ & $\mathbf{P}$ & Adjusted P \\
\hline Length of stay (days) & $27.0 \pm 15.9$ & $29.0 \pm 21.4$ & 0.411 & $<0.001$ \\
\hline $\begin{array}{l}\text { Overall intraoperative procedural com- } \\
\text { plications }\end{array}$ & $25(11.4 \%)$ & $14(21.9 \%)$ & 0.032 & 0.458 \\
\hline Thromboembolic events & $17(7.7 \%)$ & $11(17.2 \%)$ & 0.025 & 0.433 \\
\hline Hemorrhagic events & $9(4.1 \%)$ & $3(4.7 \%)$ & 0.736 & 0.813 \\
\hline Procedural cerebral infarction & $8(3.6 \%)$ & $2(3.1 \%)$ & 1.0 & 0.188 \\
\hline Ventriculostomy-related hemorrhage & & & & \\
\hline EVD & $20 / 156(12.8 \%)$ & $3 / 53(5.7 \%)$ & 0.205 & 0.212 \\
\hline VP-Shunt & $3 / 51(5.9 \%)$ & $3 / 17(17.6 \%)$ & 0.160 & 0.483 \\
\hline Overall & $22 / 157(14.0 \%)$ & $5 / 54(9.3 \%)$ & 0.367 & 0.584 \\
\hline Vasospasm & $94(42.7 \%)$ & $28(43.8 \%)$ & 0.884 & 0.002 \\
\hline Overall ischemic stroke & $64(29.1 \%)$ & $25(39.1 \%)$ & 0.130 & 0.257 \\
\hline
\end{tabular}

Table 2. Procedure-related complications. SAC, stent-assisted coiling; EVD, external ventricular drain; VP, ventriculoperitoneal.

Complications. Complications are listed in detail in Table 2. The SAC group was associated with a longer hospital stay ( $29.0 \pm 21.4$ days) than the coil group ( $27.0 \pm 15.9$ days, $\mathrm{p}=0.411$, adjusted $\mathrm{p}<0.001)$. Overall intraoperative events occurred more often during SAC than during coiling alone $(21.9 \%$ vs. $11.4 \%$, OR $2.2,95 \%$ CI $1.1-4.5, \mathrm{p}=0.032)$. After PS adjustment, this difference did not remain statistically significant (OR $1.2,95 \% \mathrm{CI}$ $0.7-2.0, \mathrm{p}=0.458$ ). In the subanalysis of complication types, SAC carried higher odds for intraoperative thromboembolic events in the unadjusted analysis (OR 2.5, 95\% CI 1.1-5.6, p = 0.025) but not in the adjusted analysis (OR 1.2, 95\% CI 0.7-2.3, p=0.433). Intraoperative hemorrhagic event rates were similar in both groups. Pro- 
cedure-related cerebral infarction were similar in the coiling (3.6\%) and the SAC group (3.1\%, p = 1.0, adjusted $\mathrm{p}=0.188$ ). There were two cerebral infarctions related to SAC. The first patient was treated by Y-stent-assisted coiling for an anterior communicating artery aneurysm. Stent deployment caused a thromboembolic occlusion of a M3 branch of the MCA. The embolus could be dissolved by tirofiban, however, postoperative CT showed a partial MCA infarction. The patient had motor dysphasia after the procedure (mRS 3). In the second case, the patient had cerebellar and mesencephalic infarction after Y-stent-assisted coiling of a basilar tip aneurysm, although no intraoperative thromboembolic events were observed. Intensive care treatment was finally discontinued in this patient (mRS 6). Overall ventriculostomy-related hemorrhage rates were $14.0 \%$ in the coiling group and $9.3 \%$ in the SAC group $(\mathrm{p}=0.367$, adjusted $\mathrm{p}=0.584)$. One patient in the coiling group required surgical evacuation of the intracranial hemorrhage after VP shunt placement. In the IPTW analysis, SAC was associated with increased odds of vasospasm (OR 1.7, 95\% CI 1.2-2.4, p=0.002), however, overall ischemic stroke rates were similar (OR 1.2, 95\% CI 0.9-1.8, $\mathrm{p}=0.257$ ).

There was no statistical difference among in-hospital mortality rates between coiling $(17.7 \%, 39 / 220)$ and SAC $(26.6 \%, 17 / 64, p=0.118$, adjusted $p=0.786)$. At discharge, $44.5 \%$ of coiled patients had a favourable mRS score, compared to $40.6 \%$ in the SAC group $(\mathrm{p}=0.578$, adjusted $\mathrm{p}=0.766)$. Among survivors, 6 -month clinical follow-up was available for $75.7 \%(137 / 181)$ in the coiling group and for $89.3 \%(42 / 47)$ in the SAC group. At 6-month follow-up, favourable outcome was achieved by $55.5 \%$ in the coiling group and by $53.1 \%$ in the SAC group $(\mathrm{p}=0.742$, adjusted $\mathrm{p}=0.471)$.

Angiographic outcome. Angiographic follow-up was available for 119 patients (54.1\%) in the coiling group and 40 patients $(62.5 \%)$ in the SAC group. Complete occlusion, neck remnants and aneurysm remnants were observed in $67.2 \%, 11.8 \%$ and $21.0 \%$ after coiling, respectively, and in $80.0 \%, 10.0 \%$ and $10.0 \%$ after SAC, respectively $(\mathrm{p}=0.361)$. After adjustment for the propensity scores, SAC had significantly higher odds for complete aneurysm occlusion (OR 3.2, 95\% CI 1.9-5.4, p < 0.001). Recanalization occurred in 17.5\% after SAC and $26.1 \%$ after coiling $(\mathrm{p}=0.273)$. After adjustment, sole coiling was significantly associated with aneurysm recurrence (OR 3.1, 95\% CI 1.7-5.4, $\mathrm{p}<0.001$ ). Retreatment rates were $22.7 \%$ for the coiling group and $17.5 \%$ for the SAC group $(\mathrm{p}=0.474)$. This difference became significant after IPTW adjustment (OR 1.7, 95\% CI 1.0-3.6, $\mathrm{p}=0.049)$.

\section{Discussion}

The results of the current study demonstrate that SAC provides superior immediate aneurysm occlusion to standalone coiling, when accounting for diverging baseline characteristics. SAC was associated with higher risks of periprocedural thromboembolic events, however, this effect was mitigated after propensity score adjustment. In this context, there were no significant differences among procedural cerebral infarction, overall ischemic stroke, in-hospital mortality, and ventriculostomy-related hemorrhage rates. A similar portion of patients in both groups achieved favourable functional outcome. SAC provided higher 6-month complete aneurysm occlusion rates, requiring retreatment less frequently than coiling alone.

Previous studies indicated that SAC carries a higher risk of thromboembolic complications than stand-alone coiling. For instance, Hetts et al. reported 1-year ischemic stroke rates of $8.8 \%$ for SAC compared to $2.2 \%$ for coiling ${ }^{3}$. In the current study, SAC had a relative risk of 2.5 for thromboembolic complications, hence supporting previous studies on mostly unruptured aneurysms. However, after propensity score adjustment, this difference was mitigated. In addition, procedure-related and overall cerebral infarction rates were comparable between both groups. Generally, SAC is performed predominantly for large, wide-necked and bifurcation aneurysms. A subanalysis of the aneurysms included in the CLARITY study demonstrated that these anatomical features represent per se an increased risk for thromboembolic complications ${ }^{12}$. However, the difference in baseline aneurysm characteristics between SAC and coiling alone is not statistically addressed by most comparative studies. Furthermore, prospective randomized studies on this topic are lacking. To our knowledge, this is the first study comparing conventional coiling and SAC using an IPTW approach, which simulates a retrospective randomization. Although IPTW cannot substitute a prospective randomized clinical trial, adjustment for the individual propensity scores allows a direct comparison of the two treatment modalities, which can be regarded as a strength of the present study. Our findings indicate that SAC has a higher risk of thromboembolic complications in clinical practice, however, this may be mainly related to the preferential treatment of morphologically complex aneurysms and not necessary by the procedure itself.

To identify a correlation between dual anti-platelet aggregation and intracranial bleeding tendency, we determined the frequency of intracranial hemorrhage after ventriculostomy. SAC patients carried a slightly higher risk of radiographic hemorrhage related to VP-shunt placement, however, this difference was not statistically significant and the hemorrhage did not require surgical evacuation in any case. In contrast, EVD-related haemorrhage occurred less frequently after SAC than after coiling, although this difference did not receive statistical significance either. Due to the necessary anti-platelet treatment related to SAC, one would expect increased ventriculostomy-related haemorrhage for these patients. Accordingly, Kung et al. revealed anti-platelet therapy as significant risk factor for both radiographic and symptomatic intracranial hemorrhage after ventriculostomy ${ }^{13}$. Darkwah-Oppong et al. confirmed an association between anti-platelets and ventriculostomy-induced bleedings, however it had no impact on functional outcome in their study ${ }^{14}$. Our institutional SAH protocol includes EVD placement before interventional aneurysm embolization and start of anti-aggregant therapy. This approach might increase the safety of ventriculostomy in case of subsequent stent implantation. Moreover, Darkwah-Oppong et al. reported that aspirin monotherapy was associated with lower odds for hemorrhagic complications than dual antiplatelet therapy ${ }^{14}$. Likewise, antiplatelet therapy did not affect functional outcome in a subanalysis of the International Subarachnoid Aneurysm Trial ${ }^{15}$. Nevertheless, most neurosurgeons would agree that restriction 
to aspirin monotherapy would be a considerable advancement for SAC of ruptured aneurysms. There are first reports on single antiplatelet therapy with coated flow-diverters ${ }^{16,17}$, however, a systematic clinical evaluation of its safety and efficacy is lacking so far and definite conclusion cannot be drawn yet.

In the current study, SAC carried increased odds of cerebral vasospasm. This result is opposed to previous findings. Andic et al. reported that SAC in the presence of vasospasm is feasible and safe and the stent can provide additional mechanical vasodilation ${ }^{18}$. Nagahama et al. reported a lower risk for vasospasm and delayed cerebral ischemia among patients receiving dual anti-platelet therapy ${ }^{19}$. Likewise, Darkwah-Oppong et al. reported a reduced risk of delayed cerebral ischemica and more favourable outcome among SAH patients under aspirin therapy ${ }^{20}$. In our study, the cerebral infarction rates were comparable between SAC and coiling and a similar portion of patients achieved favourable clinical outcome in both groups. These findings are in line with previous studies. Zhang et al. conducted a meta-analysis that included eight retrospective studies comparing SAC and stand-alone coiling of ruptured aneurysms. The authors reported increased rates of hemorrhagic (OR 1.6, $95 \%$ CI 1.1-2.4, $\mathrm{p}=0.319$ ) and thromboembolic events (OR 1.8, 95\% CI 1.3-2.4, p = 0.511) in the SAC group, however, the favourable clinical outcome rates was similar in both groups (OR 0.95, 95\% CI 0.88-1.02, $\mathrm{p}=0.338$ ). Summarizing these results, anti-platelet medication after SAC might slightly increase the risk of intracranial bleeding, however, these are mostly minor incidents associated with low morbidity.

Numerous studies have shown higher complete occlusion and lower recurrence rates of SAC compared to coiling without stent-assistance ${ }^{3,4,21,22}$. Jahshan et al. reported complete aneurysm occlusion in $64.6 \%$ after SAC compared to $49.7 \%$ after coiling alone ${ }^{22}$. Piotin et al. described aneurysm recanalization in $14.9 \%$ after SAC compared to $33.5 \%$ after sole coiling ${ }^{4}$. In the meta-analysis on ruptured aneurysms by Zhang et al., the immediate complete occlusion rate in the SAC groups was significantly lower than that in the non-SAC group (54.3\% vs. $64.2 \%$, OR $0.90,95 \%$ CI $0.83-0.99)^{11}$. However, SAC achieved a mid-term occlusion rate of $73.4 \%$ compared to $61.0 \%$ of stand-alone coiling (OR 1.30, 95\% CI 1.16-1.46). Likewise, recanalization rates of SAC were lower (4.8\% vs. $16.6 \%$, OR $0.28,95 \%$ CI $0.16-0.50)$. The 6-month angiographic results in our series are within the range cited by Zhang et al. ${ }^{11}$. In particular, the superior immediate and 6-month aneurysm occlusion of SAC becomes evident in the adjusted analysis which accounts for the comparably complex aneurysm morphology of SAC-treated aneurysms. A large aneurysm size and neck width have been identified as risk factors for incomplete aneurysm occlusion and recanalization, both for stent-assisted coiling ${ }^{23}$ and stand-alone coiling ${ }^{24}$. A high packing density and immediate complete occlusion are well-known requisites to minimize the risk of recanalization ${ }^{24-26}$. Intracranial stents serve as scaffold for neo-endothelialisation and prevent protrusion of the implanted coils which facilitates a dense coil packing promoting progressive and durable aneurysm occlusion.

Taking into account both clinical and angiographic outcome, the results of the meta-analysis by Zhang et al. and our data indicate that SAC of large and wide-necked ruptured aneurysms is safe and effective, providing better aneurysm occlusion and similar clinical outcome to stand-alone coiling ${ }^{11}$. In line, Tähtinen et al. recommended explicitly the use of SAC for morphologically complex ruptured aneurysms 9 . A large prospective randomized clinical trial would be ultimately required to provide definitive information on the best therapeutic approach for endovascular treatment of ruptured intracranial aneurysms.

Besides conventional stents, novel stent-like neck bridging devices have been established for treatment of ruptured intracranial aneurysms. For the pCONus (Phenox, Bochum, Germany), a laser-cut stent with a distal crown which is placed inside the aneurysm, Aguilar Pérez et al. reported intraprocedural complications in $9.5 \%$ and $62 \%$ achieved a good functional outcome. The complete occlusion rate was $56 \%$ and the recurrence rate was $25 \%{ }^{27}$. For the successor version, the pCONus HPC, the authors suggested the potential used of single antiplatelet therapy ${ }^{28}$. Other neck bridging devices, such as the endovascular clip system (eCLIPs, Evasc Medical Systems, Vancouver, Canada ${ }^{29}$ and the PulseRider (Johnson \& Johnson, New Brunswick, NJ, USA) ${ }^{30}$ showed an adequate safety and efficacy profile in preliminary studies, but have been mainly studied for unruptured aneurysms. These devices may represent a treatment alternative for selected aneurysms, especially for wide-necked and bifurcation aneurysms. For devices with a reduced metal surface, single antiplatelet therapy might suffice, which could further increase the safety of stent-assisted procedures.

The limitations of this study are mainly related to its retrospective design and the moderate number of included patients. Although the performed IPTW analysis enhances the validity of our results, we cannot exclude a potential selection bias. Moreover, clinical outcome was not determined systematically but retrospectively based on the neurological descriptions in the medical charts. The angiographic follow-up rate was only 56\% (159/284), which may not reflect the real angiographic results. Furthermore, few patients treated by coiling alone underwent follow-up by CTA and MRA, which might impede imaging analysis. In this context, aneurysm occlusion was not determined by a core laboratory which might bias the interpretation of the angiographic results ${ }^{31}$. Finally, we did not report long-term outcome, however, it can be expected that the differences in aneurysm occlusion between SAC and stand-alone coiling might become more evident at long-term follow-up.

\section{Conclusions}

The results of this IPTW-adjusted analysis indicate that SAC of ruptured intracranial aneurysms can provide improved angiographic results with no additional morbidity when compared to stand-alone coiling in carefully selected cases. Moreover, the results indicate that the risks of thromboembolic and hemorrhagic events are mainly related to the aneurysm morphology and not to the treatment modality. The introduction of novel devices with reduced metal surface and potential single antiplatelet therapy may further increase the safety of $\mathrm{SAC}$ in the future. 


\section{Methods}

The study protocol was approved by the local ethics committee of the University Hospital of Cologne (IRB 13-104). The need for informed consent was waived by the local ethics committee of the University Hospital of Cologne. The study was conducted in accordance with the STROBE guidelines in compliance with the national legislation and the Code of Ethical Principles for Medical Research Involving Human Subjects of the World Medical Association (Declaration of Helsinki).

Inclusion and exclusion criteria. Consecutive SAH patients treated at a single center between January 2010 and December 2019 were retrospectively reviewed. All patients that underwent endovascular treatment for an acutely ruptured intracranial aneurysm within 14 days after ictus were considered. This time period was selected, because patients were surveilled at the intensive care unit for at least 14 days after ictus. Patients treated with coiling only, balloon-assisted coiling and stent-assisted coiling were included. Exclusion criteria were: (1) Microsurgical clipping, (2) flow-diverter implantation (3) treatment with the Woven Endobridge (WEB), (4) parent artery occlusion, (5) recurrent aneurysms, (6) partially thrombosed aneurysms, (7) fusiform aneurysms, and (8) dissecting aneurysms. Patients treated by coiling only and balloon-assisted coiling were subsumed in the "coiling" group.

Procedure. All procedures were performed via a transfemoral approach with the patient under general anesthesia in a biplane angiosuite (Philips, Best, the Netherlands). Intracranial stenting was performed using a Headway 17 (Microvention, Tustin, CA, USA) or Prowler Select Plus (Codman Neurovascular, Raynham, MA, USA) microcatheter depending on the individual microstent type. The following stent types were employed: Acclino (Acandis, Pforzheim, Germany), Solitaire AB (Medtronic, Dublin, Ireland), Enterprise (Johnson \& Johnson, New Brunswick, NJ, USA), Neuroform Atlas (Stryker, Kalamazoo, MI, USA), and LVIS jr (Microvention, Tustin, CA, USA). The adjunctive use of stents, stent type and number of stents was left to the discretion of the neurointerventionalist. The standard approach of SAC consisted of stent deployment across the aneurysm neck, followed by probing the aneurysm sac with a microcatheter through the stent interstices and final coil embolization. Furthermore, the microcatheter jailing technique was used, in which the stent is deployed after microcatheterization of the aneurysm sac by an additional microcatheter and before coil deployment. Bifurcation aneurysms were treated either with a single stent which is placed across the branching vessels or with two stents using the Y-stent technique.

In case of intra-procedural re-rupture, the rupture site was immediately embolized with coils to stop the bleeding. Therafter, the aneurysm was treated as originally planned. After the procedure, a CT scan was performed to determine the extent of re-hemorrhage. In case of intra-procedural thromboembolism, a loading dose of intra-arterial tirofiban (infusion rate: $0.4 \mu \mathrm{g} / \mathrm{kg} / \mathrm{min}$ ) was applied for $30 \mathrm{~min}$. In some cases additional mechanical thrombectomy was performed. A control CT scan with perfusion sequences was performed to evaluate cerebral infarction.

Angiographic follow-up was performed 6 months after the procedure using DSA in the majority of cases. In few patients treated by coiling alone, magnetic resonance angiography and computed tomography angiography was used instead of DSA. The Raymond-Roy occlusion classification (RROC) was used to evaluate aneurysm occlusion: 1, complete occlusion, 2, neck remnant, and 3, aneurysm remnant. Aneurysm recurrence was defined as an increase of the RROC score at follow-up compared to post-treatment. Upon proof of aneurysm remnants, the need and modality for retreatment was discussed within an interdisciplinary neurovascular board.

Anti-aggregation therapy. I.v. Heparin was not administered routinely. In case of adjunctive stent deployment, tirofiban (Aggrastat, Merck, West Point, PY, USA) was applied weight adapted according to the manufacturer's guidelines, starting promptly before stent placement and continued for $16-24 \mathrm{~h}$ after the procedure. Tirofiban is given intravenously at an initial infusion rate of $0.4 \mu \mathrm{g} / \mathrm{kg} / \mathrm{min}$ for $30 \mathrm{~min}$. At the end of the initial infusion, tirofiban is continued at a maintenance infusion rate of $0.1 \mu \mathrm{g} / \mathrm{kg} / \mathrm{min}$. Thereafter, the patients were loaded with $500 \mathrm{mg}$ acetylsalicylic acid (ASA) and $300 \mathrm{mg}$ clopidogrel. Maintenance anti-platelet therapy consisted of ASS $100 \mathrm{mg} /$ day life-long and clopidogrel $75 \mathrm{mg} /$ day.

Data collection and definition of outcome parameters. Medical charts and operation records were retrospectively reviewed to determine the following parameters: patient age, sex, World Federation of Neurosurgical Societies (WFNS) grading scale, Fisher scale, time interval between ictus and treatment, length of hospital stay, procedural adverse events, in-hospital mortality, external ventricular drain (EVD)/ventriculoperitoneal shunt placement and neurological status at follow-up. Procedural adverse events include intraoperative thromboembolic events (e.g. in-stent stenosis, thromboembolism) and intraoperative hemorrhagic events (e.g. aneurysm perforation, rebleeding) independently of their clinical significance. Native CT scans and CT angiography were reviewed in order to determine vasospasm and cerebral infarction. Procedure-related infarction was defined to be spatially and temporally related to the procedure without the occurrence of concomitant vasospasm. Furthermore, 30-day overall ischemic stroke rates (including procedure-related infarction and delayed cerebral ischemia) are reported. In patients that underwent EVD and/or VP-shunt placement, postoperative CT scans were reviewed to identify ventriculostomy-related hemorrhage, which was defined as a new intraparenchymal bleeding along the ventricular catheter tract. Functional outcome was assessed at 6-month follow-up determining the modified Rankin scale (mRS) score on the basis of the neurological status. Patients that were not available for 6-month follow-up visits were contacted by phone. A mRS score $\leq 2$ was defined as favourable outcome and a mRS score $>2$ as unfavourable outcome, whereby a mRS score of 6 defines death. Four-vessel 
digital subtraction angiography (DSA) scans were reviewed to determine aneurysm size, neck width, and dometo-neck $(\mathrm{D} / \mathrm{N})$ ratio.

Favourable functional outcome at 6-month follow-up was defined as primary outcome measure. Secondary outcome measures were intraoperative thromboembolic and hemorrhagic events, immediate complete occlusion, procedure-related and overall cerebral infarction, in-hospital mortality, ventriculostomy-related haemorrhage, 6-month complete occlusion and retreatment.

Statistical analysis. Qualitative data are presented as numbers and percentages. Groups are compared using the Chi-Square and the Fisher exact text, when appropriate. Quantitative data are presented as means and standard deviation and compared using the unpaired t-test and the Mann-Whitney U test. Normal distribution of quantitative parameters was evaluated with the Shapiro-Wilk test. Inverse probability of treatment weighting (IPTW) based on individual propensity scores was applied to counteract a potential selection bias and to control for differences in baseline characteristics between the coiling alone and the SAC group. This methods allows a retrospective randomization. An individual propensity score was calculated for each patient as the predicted probability for SAC by using a multivariate logistic regression model with stent-assisted treatment as the response and the following covariates: patient age, sex, WFNS grade, Fisher grade, aneurysm location, aneurysm size, and neck width. In the IPTW method, each patient was weighted by the inverse probability of being in either the coiling alone or the SAC group. This approach leads to the creation of two synthetic study groups which have similar propensity scores and thus comparable baseline characteristics. Statistical analysis was performed using SPSS software (IBM SPSS Statistics for Windows, Version 25.0, Armonk, NY, USA). A p-value $<0.05$ was considered as statistically significant. For propensity score analysis, the R 3.3.0 plug-in (https://cran.r-project.org/) was installed.

\section{Data availability}

All data will be made available upon request in an anonymized manner.

Received: 23 June 2021; Accepted: 14 October 2021

Published online: 05 November 2021

\section{References}

1. Goertz, L. et al. Safety and efficacy of the Neuroform Atlas for stent-assisted coiling of intracranial aneurysms: A multicenter experience. J. Clin. Neurosci. 68, 86-91 (2019).

2. Goertz, L. et al. Long-term angiographic results of the low-profile acandis acclino stent for treatment of intracranial aneurysms. Clin. Neuroradiol. 30, 827-834 (2019).

3. Hetts, S. et al. Stent-assisted coiling versus coiling alone in unruptured intracranial aneurysms in the matrix and platinum science trial: Safety, efficacy, and mid-term outcomes. Am. J. Neuroradiol. 35, 698-705 (2014).

4. Piotin, M. et al. Stent-assisted coiling of intracranial aneurysms: Clinical and angiographic results in 216 consecutive aneurysms. Stroke 41, 110-115 (2010).

5. Goertz, L. et al. Low-profile laser-cut stents for endovascular treatment of intracranial aneurysms. Clin. Neuroradiol. 31, 107-115 (2020).

6. Mahaney, K. B. et al. Risk of hemorrhagic complication associated with ventriculoperitoneal shunt placement in aneurysmal subarachnoid hemorrhage patients on dual antiplatelet therapy. J. Neurosurg. 119, 937-942 (2013).

7. Tumialán, L. M. et al. Intracranial hemorrhage associated with stent-assisted coil embolization of cerebral aneurysms: A cautionary report. J Neurosurg 108(6), 1122-1129 (2008).

8. Goertz, L. et al. Safety and efficacy of the derivo embolization device for the treatment of ruptured intracranial aneurysms. J. NeuroInterv. Surg. 11, 290-295 (2019).

9. Tähtinen, O. I. et al. Wide-necked intracranial aneurysms: Treatment with stent-assisted coil embolization during acute ( $<72 \mathrm{~h})$ subarachnoid hemorrhage-Experience in 61 consecutive patients. Radiology 253, 199-208 (2009).

10. Yang, P. et al. Stent-assisted coil placement for the treatment of 211 acutely ruptured wide-necked intracranial aneurysms: A single-center 11-year experience. Radiology 276, 545-552 (2015).

11. Zhang, X. et al. Stent assisted coiling versus non-stent assisted coiling for the management of ruptured intracranial aneurysms: A meta-analysis and systematic review. J. NeuroInterv. Surg. 11, 489-496 (2019).

12. Pierot, L., Cognard, C., Anxionnat, R., Ricolfi, F. \& Investigators, C. Ruptured intracranial aneurysms: Factors affecting the rate and outcome of endovascular treatment complications in a series of 782 patients (CLARITY study). Radiology 256, 916-923 (2010).

13. Kung, D. K. et al. Risk of ventriculostomy-related hemorrhage in patients with acutely ruptured aneurysms treated using stentassisted coiling. J. Neurosurg. 114, 1021-1027 (2011).

14. Oppong, M. D. et al. Secondary hemorrhagic complications in aneurysmal subarachnoid hemorrhage: When the impact hits hard. J. Neurosurg. 132, 79-86 (2019).

15. van den Bergh, W. M., Kerr, R. S., Algra, A., Rinkel, G. J. \& Molyneux, A. J. Effect of antiplatelet therapy for endovascular coiling in aneurysmal subarachnoid hemorrhage. Stroke 40, 1969-1972 (2009).

16. Bhogal, P. et al. The p48_HPC antithrombogenic flow diverter: Initial human experience using single antiplatelet therapy. J. Int. Med. Res. 48, 0300060519879580 (2020).

17. Hanel, R. A., Aguilar-Salinas, P., Brasiliense, L. B. \& Sauvageau, E. First US experience with pipeline flex with shield technology using aspirin as antiplatelet monotherapy. Case Rep. https://doi.org/10.1136/bcr-2017-219406 (2017).

18. Andic, C., Kardes, O., Durdag, E., Gedikoglu, M. \& Akin, S. Efficacy of endovascular treatment and feasibility of stent-assisted coiling in the presence of severe and symptomatic vasospasm. J. NeuroInterv. Surg. 9, 1075-1080 (2017).

19. Nagahama, Y. et al. Dual antiplatelet therapy in aneurysmal subarachnoid hemorrhage: Association with reduced risk of clinical vasospasm and delayed cerebral ischemia. J. Neurosurg. 129, 702-710 (2017).

20. Darkwah Oppong, M. et al. Post-treatment antiplatelet therapy reduces risk for delayed cerebral ischemia due to aneurysmal subarachnoid hemorrhage. Neurosurgery 85, 827-833 (2019).

21. Hong, Y., Wang, Y.-J., Deng, Z., Wu, Q. \& Zhang, J.-M. Stent-assisted coiling versus coiling in treatment of intracranial aneurysm: A systematic review and meta-analysis. PLoS ONE 9, e82311 (2014).

22. Jahshan, S. et al. Results of stent-assisted vs non-stent-assisted endovascular therapies in 489 cerebral aneurysms: Single-center experience. Neurosurgery 72, 232-239 (2012). 
23. Chalouhi, N. et al. Stent-assisted coiling of intracranial aneurysms: Predictors of complications, recanalization, and outcome in 508 cases. Stroke 44, 1348-1353 (2013).

24. Murayama, Y. et al. Guglielmi detachable coil embolization of cerebral aneurysms: 11 years' experience. J. Neurosurg. 98, 959-966 (2003).

25. Raymond, J. et al. Long-term angiographic recurrences after selective endovascular treatment of aneurysms with detachable coils. Stroke 34, 1398-1403 (2003).

26. Sadato, A., Hayakawa, M., Adachi, K., Nakahara, I. \& Hirose, Y. Large residual volume, not low packing density, is the most influential risk factor for recanalization after coil embolization of cerebral aneurysms. PLoS ONE 11, e0155062 (2016).

27. Pérez, M. A. et al. Use of the pCONus as an adjunct to coil embolization of acutely ruptured aneurysms. J. NeuroInterv. Surg. 9, 39-44 (2017).

28. Perez, M. A. et al. Use of the pCONus HPC as an adjunct to coil occlusion of acutely ruptured aneurysms: Early clinical experience using single antiplatelet therapy. J. NeuroInterv. Surg. 12, 862-868 (2020).

29. De Vries, J. et al. eCLIPs bifurcation remodeling system for treatment of wide neck bifurcation aneurysms with extremely low dome-to-neck and aspect ratios: A multicenter experience. J. NeuroInterv. Surg. 13, 438-442 (2021).

30. Spiotta, A. M., Chaudry, M. I., Turk, A. S. \& Turner, R. D. Initial experience with the PulseRider for the treatment of bifurcation aneurysms: Report of first three cases in the USA. J. NeuroInterv. Surg. 8, 186-189 (2016).

31. Rezek, I. et al. Differences in the angiographic evaluation of coiled cerebral aneurysms between a core laboratory reader and operators: Results of the Cerecyte Coil Trial. Am. J. Neuroradiol. 35, 124-127 (2014)

\section{Author contributions}

L.G., T.L., L.P., H.S., M.T., J.P.G. and M.S. and acquired the data. L.G. and C.K. developed the project. L.G., T.L. and C.K. interpreted the data. L.G. and C.K. drafted the manuscript. All authors revised the paper critically for important intellectual content and provided final approval of the version published. All authors agree to be accountable for all aspects of the work in ensuring that questions related to the accuracy or integrity of any part of the work are appropriately investigated and resolved.

\section{Funding}

Open Access funding enabled and organized by Projekt DEAL.

\section{Competing interests}

CK serves as consultant for Acandis GmbH (Pforzheim, Germany) and as proctor for MicroVention Inc./Sequent Medical (Aliso Viejo, CA, USA). TL serves as proctor for MicroVention Inc./Sequent Medical (Aliso Viejo, CA, USA), CERUS Endovascular (Fremont, CA, USA), Phenox (Bochum, Germany), Stryker (Kalamazoo, MI, USA), and Medtronic (Dublin, Ireland). The other authors declare that they have no competing interests.

\section{Additional information}

Correspondence and requests for materials should be addressed to L.G.

Reprints and permissions information is available at www.nature.com/reprints.

Publisher's note Springer Nature remains neutral with regard to jurisdictional claims in published maps and institutional affiliations.

(c) (i) Open Access This article is licensed under a Creative Commons Attribution 4.0 International License, which permits use, sharing, adaptation, distribution and reproduction in any medium or format, as long as you give appropriate credit to the original author(s) and the source, provide a link to the Creative Commons licence, and indicate if changes were made. The images or other third party material in this article are included in the article's Creative Commons licence, unless indicated otherwise in a credit line to the material. If material is not included in the article's Creative Commons licence and your intended use is not permitted by statutory regulation or exceeds the permitted use, you will need to obtain permission directly from the copyright holder. To view a copy of this licence, visit http://creativecommons.org/licenses/by/4.0/.

(c) The Author(s) 2021 